the average amount. He had control over the sphincters of the bladder and rectum. The knee-jerks and plantar reflexes were lost. The pain in his back ceased after twelve hours and next day he felt much better and he ras able to move his legs freely. On the following morning he was able to walk a few steps and he expressed himself as feeling almost
quite well. At this period I showed him to the hospital class and I quite well. At this period I showed him to the hospital class and I
diagnosed the case as plumbism and chronic interstitial nephritis, with the usual cardio-vascular changes. I pointed out that the history of the attack clearly indicated some vascular lesion of the spinal cord, and the surden onset. widle diffusion, and intensity of the pain, together with the transient duration of the paraplegia, appeared to justify the further diagnosis of spinal meningeal hæmorrhage. The patient was apparently well about $40^{\circ}$ clock in the afternoon, when he took a drink of mill. Fire minutes afterwards the nurse found him dead in his bed. symptoms.

The post-mortem examination, which was made within 24 hours of his death. showed the right pleural cavity to be filled with blood. The pericardium contained a little clear serous fluid. The heart was rather large, there being hypertrophy of the left ventricle. The arch and upper part of the descending aorta was distended and formed a tumourlike mass, from which the blood had escaped into the right pleura. On opening the aorta its inner aspect was corered with atheromatous plaques, and a dissecting aneurysm extended from the arch at the level of the innominate artery and terminated in the left femoral artery about an inch below Poupart's ligament. A large mass of freshlyabout an inch below Poupart's ligament. A large mass of freshly and in the upper portion of the descending thoracic aorta, and here the and in the upper portion of the descending thoracic aorta, and here the separation of the tunics took place around nearly the whole circum-
ference of the vessel wall. From the abdominal aorta through the left common illace external iliac, and femoral artery the coats of the vessels we to salp thickening of the arteries dissection of the arterial coats did not extend much more than halfway around the circumference of the vessels.

The microscopic sections, which I exhibited, were from theleft common iliac artery, and they showed that the blood was effused in the substance of the midale tunic of the artery, so that the external wall of the aneurysmal canal was composed of the outer muscular strata of the
media together with the adventitia. The dissection was carried along media together with the adventitia. The dissection was carried along by a thrombus. The lumbar arteries were cut across and their orifice likewise contained thrombi. A large coagulum was adherent to the posterior wall of the aorta at the level of the renal and lumbar arteries. The external rupture of the aneurysm was situated about th middle of the thoracic aorta on its posterior aspect. The rupture was an ill-defined, oblique slit, about half an inch long, from which the blood had forced its way through the connective tissue into the right pleura. I have not been able to satisfy myself as to the position of the internal rupture. It might have occurred two or three inches from the termination of the abdominal aorta, for the atheromatous changes were most intense in that position or possibly it took place at the origin of one of the primary branches of the aorta. Both kidneys were cirrhotic the right one being in addition, cystic and extremely engorged with blood Microscopic sections of this kidney (prepared by Dr. Dargan in Dr. MeWeener's laboratory) showed that it was in a condition of universal hæmorrhagic infarction. There was no hæmorrhage in the spinal cord or its membranes. In the light of the necropsy it was not difficult to account for the symptoms of this case. As prestisposing causes of dissecting aneurysm my patient had extensive atheroma of the inner arldition he had hypertrophy of the left ventricle with high arterial arldition he had hypertrophy of the left ventricle with high arterial
tension. The intense pain at onset of the symptoms was due to the cension. The intense pain at onset of the symptoms was due to the the palaplegia was caused by the interference with the arterial supply the palaplegia was caused by the interference with the arterial supply to the lumbar enlargement of the cord, partly from thrombosis, and partly from rupture of the lumbar arteries; the sudden death ensued on the rupture of the external wall of the aneurysm and the consequent
escape of blood into the right pleura. It is rather remarkable that his urine contained no blood and that the amount passed was not noticeably diminished, for the right kidney was engorged with blood and its ecretion must have been completely arrested.

Other cases of dissecting aneurysm have been recorded in which a remarkable series of symptoms was due to arrest of vascular supply to the brain, kidneys, or cord. Tessier's ${ }^{17}$ patient died with the symptoms of apoplexy and a dissecting aneurysm was found extending from the arch of the aorta along the innominate artery and its branches to the internal carotid. Todd's ${ }^{18}$ case was characterised by hemiplegia and transient suppression of urine, and the post-mortem examination showed softening of the brain due to obstruction in the carotids, and it is probable that there was also obstruction in the renal arteries. Sainet ${ }^{19}$ records a case in which the patient became rapidly paraplegic. Latham and Swaine's ${ }^{20}$ case was diagnosed during life. The patient was suddenly seized with "agonising" pain in his chest followed by paraplecia. In Dickenson's 21 case a policeman, after seven hours on his beat, was seized with loss of power over his lower extremities, follower by pain and collapse. Death took place within twenty-four hours. Where the symptoms are liable to such variations it is evident that the diagnosis of dissecting aneurysm must always be difficult and uncertain.

In reference to treatment Walshe ${ }^{22}$ somewhat cynically

17 Giornale della Scienza Mediche, 1842

18 Transactions of the Royal Medical and Chirurgical Society

₹ol. xxvii.. 1844.

20 Transactions of the Pathological Society of London, vol. vii., 1855."

22 Walshe: Diseases of theif Heart. remarks: "Were the practitioner fortunate enough to divine the occurrence of acute separation of the coats of the aorta it does not appear that in the present state of our knowledge the management of the case would be materially improved by his sagacity. Did he fail to diagnose the occurrence his aim would be to restore the patient from the first shock of the accident, control excited arterial action, and relieve symptoms as they arose. And it does not appear that art could do more than this were the anatomical nature of the affection understood from the first."

Dublin.

\section{ON A MODE OF STRETCHING SOME URETHRAL STRICTURES.}

BY REGINALD HARRISON, F.R.C.S. ENG., SURGEOY TO ST. PETER'S HOSPITAL.

IN some remarks on urethral stricture previously published ${ }^{1}$ I have referred more particularly to its treatment by dilatation and by certain forms of urethrotomy or section. I have also described some instruments I usually employ with these processes. In an obstructive disease which presents so much structural variety cases are occasionally met with where, either from the urgency of the symptoms or the nature of the stricture, it will be found impossible to utilise these methods and modifications of them may be substituted with advantage.

The rarer cases of advanced stricture to which I am now referring usually owe their urgency to being complicated with some degree of retention of urine which also calls for surgical aid. A man of middle age, I will say, has been suffering from a neglected stricture for some months or years. Eventually he is either suddenly seized with inability to pass urine at all or he becomes seriously inconvenienced by the increased tension of a never emptied bladder. Let it be assumed that the stricture is so hard, contracted, or tortuous as to resist hot baths, opium, and catheterism with all forms of flexible instruments, and access to the bladder can only be effected by a fine metallic catheter. Possibly this even may require the aid of an anæsthetic. If such a catheter is tied in it not un. frequently slips out during the night and all the trouble of replacing it or substituting a somewhat larger one has to be repeated and not always with success. Even if it is retained it may not be possible to substitute a larger one and thus the progress of the case in giving increased relief to the patient is either hindered or proves impossible. Nor is the retention of a fine catheter, such as No. 1, 2, or 3, in a septic bladder to be desired, even though some relief to tension is afforded, any more than a drainage tube which will not drain is of service with a putrid abscess. Unless the relief is sufficient in either case sepsis is sure to follow if it has not already commenced. With a small catheter such as those I have referred to it is impossible to wash out and disinfect the bladder, and puncture or any forms of cystotomy or urethrotomy are not always advisable under these circumstances. It is in cases of this type, when the surgeon has succeeded in passing a small metallic catheter through a tight and difficult stricture into a more or less distended bladder, that the inability to expand the instrument at the same time, and so to stretch the stricture before withdrawing it, is not unfrequently experienced. With this object in view I described some years ago an instrument constructed on the lines of a Holt' dilator which appeared to meet the difficulty, and I have since continued to use it with advantage. It is provided with a pilot guide as well as with a screw top in case the former proves useless. It contains a fine test catheter on which the dilators run. It is fitted with a series of seven rods by which dilatation may be carried from a No. 3 to a No. 12 (English gauge), and the introduction of the latter is controlled by a spiral spring which consolidates the instrument and makes it act as one piece. On the dilator being passed, as an equivalent to a No. 3 catheter, the stylet is withdrawn and the correct position of the instrument is judged of by its transmitting urine and from its position relative to the rectum. Then the introduction of the dilating rods in accordance with their number and size is 
proceeded with whilst the anæsthetic is continued. This is to normal dimensions of his urethra. It is hardly necessary to be done deliberately so as to stretch and not to lacerate the illustrate the kind of case to which this treatment is applicstricture, the process usually lasting, according to the rigidity able, as it will readily occur to those who hare had ant of the contraction, from about ten to twenty minutes. When practical experience of them. In proceeding to adopt it the the largest rod is reached the fully distended instrument, now only drawback which is likely to happen is the case proving. representing No. 12 in calibre, is quietly withdrawn and the on trial with an anæsthetic, to be an impassable stricture. urine that remains is drawn off by a full-sized silver catheter, Under such circumstances Wheelhouse's perineal operation

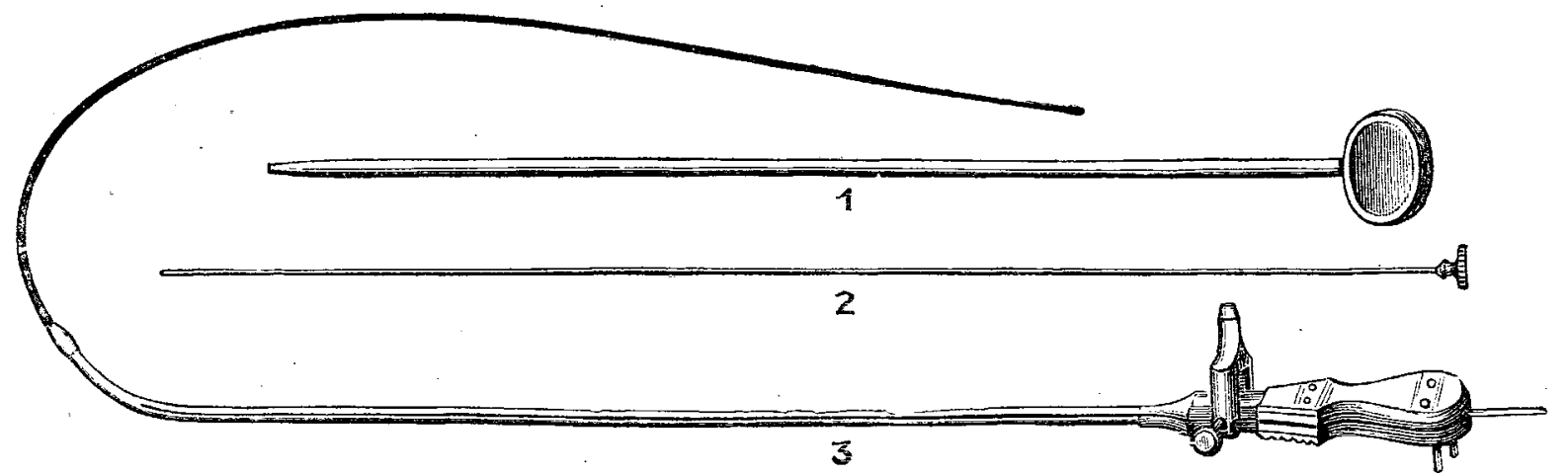

Figures illustrating instrument, made by Messrs. Krohne and Sesemann. 1, A dilating rod. 2, Catheter stylet. 3, Dilator.

which may be passed easily. The bladder is then washed out and disinfected and a soft catheter is tied in and retained for forty-eight hours or so. As the process is one of stretching alone rigors or fever rarely follow.

The subsequent management of the case merely consists in instructing the patient how and when to pass a bougie for himself and so to endeavour to permanently maintain the

\section{ON A MODIFICATION OF MR. COXONS' METHOD OF PROLONGING NITROUS OXIDE AN ÆSTHESIA DURING DENTAL OPERATIONS- \\ NAMELY, BY MEANS OF A MOUTH TUBE AND CLOSURE} OF THE NARES.

BY W. J. McCARDIE, B.A., M.B., B.C. CANTAB., ANESTHETIST TO THE GENERAL HOSPITAL AND TO THE DENTAL HOSPITAI, BIRMINGHAM.

THE recent endeavours to prolong nitrous oxide anæsthesia for the purposes of dental surgery by means of a mouth-tube, nasal catheter, or nose-piece have all tended to supply a very distinct need-viz., an anæsthesia longer than the usual one of about half a minute and perhaps not so long a one as that obtained so easily and safely by the administration of nitrous oxide followed by ether.

I believe that for all lengthy or severe dental operations, especially in cases where numerous teeth or many bad stumps have to be extracted or in cases in which the patient is very young or old or in feeble health and the operation is of more than ordinary severity, three or four full breaths of nitrous oxide followed by a short inhalation of ether is by far the best method of anæsthetisation, for the shock during and after operation is in these cases less than that occurring during or after the administration of gas. The delayed return of consciousness is often of distinct advantage to both the operator and the patient, and the anæsthetist is left with a freer hand to help the dentist, who for his part can with confidence reckon on having plenty of time and is not embarrassed perhaps with anæsthetic appliances.

Last winter, at a meeting of the Society of Anæsthetists, Mr. Coxon, L.D.S., of Wisbech, demonstrated his apparatus for the prolongation of nitrous oxide anæsthesia. It consisted essentially of a mouth-tube made of hard metal with a diameter of about half an inch, at one end nearly half curved and at the other made to fit in the place of the face-piece and stopcock of an ordinary gas apparatus. Mr. Coxon warmed the gas before use by passing it through a metal coil immersed in hot water and interposed between the gas cylinders and the gas bag. In administration the patient was in the usual way put fully under the influence of nitrous oxide, then the facepiece was rapidly replaced by the oral tube, which was inserted into the mouth in such a way that it rested on the back of the tongue, and the free end was in the pharynx pointing towards the side of that cavity. The anæsthetist had of course to keep the tube out of the way of the would probably be selected as the alternative. Where, however, the dilator can be passed in the manner I have endeavoured to describe both patient and practitioner will be none the worse for knowing that the retention of urine has been relieved in such a way as will permit the introduction of a full-sized catheter into the bladder.

Lower Berkeley-street, W.

operator and to arrange that a copious stream of gas was delivered from a fully-distended gas bag and passed on to the pharynx beyond the region involved in the operation and also beyond any collection of blood in the mouth. Mr. Coxon stated that he had often used this method of his devising and had had exceedingly good results from it.

I was much interested in his demonstration and had a metal tube like his made for me, but not regarding the warming of the gas as of importance in short operations at any rate, even if it be so in long operations, which $I$ think doubtful, I made trial of the method without using any heating apparatus on many patients at the Birmingham Dental Hospital. As I preferred to keep the fullest command of the head and jaws and had to manipulate the gag $I$ asked Mr. Thompson Madin, L.D.S., surgeon to the hospital, to kindly insert the mouth-tube for me and take charge of the gas-bag while I controlled the anæsthesia by means of the gas-supply.

The first few cases of trial were not very successful, but we soon became more proficient, and after a little time the great difficulty - that of inserting the tube to the back of the mouth and at the same time keeping it out of the way of the operator, especially in cases where the mouth was smallwas dexterously overcome. We found that a central gag-e.g., Brunton's-wherever it could be used was the best. The gas-bag had generally to be kept fully distended and in the case of strong patients even had to be compressed between the arm and side of the body to increase the delivery pressure. It will be seen that a good deal of gas had to be used, for it escaped both by the mouth and the nose. I constantly for some months used this method in cases which needed a longer administration than usual and had a large measure of success. In many instances the duration of the anæsthesia was only determined by the conclusion of the operation. As the extractions were performed by students much longer periods of anæsthesia than usual were sometimes necessary and times of from one to four minutes were recorded without any untoward present or after-effects being noticed except that giddiness lasted perhaps a little time longer than after the ordinary sitting. I judged of the degree of anæsthesia chiefly by the colour of the face, managing the supply of gas so that the patient's lips were kept slightly cyanosed and the breathing rather snoring in character. After the above method had been in use for a few months Mr. Madin ingeniously thought of the modification of closing the nasal passages in order to prevent the escape of nitrous oxide and to limit air entry. After closing the nose by manual pressure he proposed to pass the tube just inside the mouth at a corner and to keep it full of gas by using only a moderate pressure. He himself made trial of this plan with perfect success. As the closure of the nostrils by digital compression 